\title{
Highly Enantioselective Catalytic Phenylation of Ketones with a Constrained Geometry Titanium Catalyst
}

\author{
Celina García and Patrick J. Walsh* \\ P. Roy and Diane T. Vagelos Laboratories, \\ University of Pennsylvania, Department of Chemistry \\ 231 South $34^{\text {th }}$ Street, Philadelphia, PA 19104-6323.
}

\section{Supporting Information}

Table of Contents

page

General Methods

S2

General Procedure for the Preparation of Tertiary Alcohols

S2

Characterization of $\mathbf{S 1}-\mathbf{S 1 1}$

S2

Conditions for the Determination of Enantiomeric Excess

S7 
General Methods. All reactions using diphenylzinc and titanium(IV) isopropoxide were carried out in a Vacuum Atmospheres dry box, with an attached MO-40 Dritrain, or by using standard Schlenk and vacuum line techniques with ovendried glassware. All chemicals were obtained from Acros Organics or Aldrich. All solvents were purchased from Fischer Scientific. Diphenylzinc was purchased from Strem and stored as a solid in a Vacuum Atmospheres dry box. All liquid ketone substrates were distilled prior to use. Toluene and hexanes were dried through alumina columns. The progress of all reactions was monitored by thin-layer chromatography to ensure the reactions had reached completion. ${ }^{1} \mathrm{H}$ NMR and ${ }^{13} \mathrm{C}$ NMR spectra were obtained on a Brüker AM-500 Fourier transform NMR spectrometer at 500 and 125 $\mathrm{MHz}$, respectively. Chemical shifts are reported in units of parts per million downfield from tetramethylsilane, and all coupling constants are reported in Hertz. Silica gel (230400 mesh, Silicycle) was used for air-flashed chromatography. Thin-layer chromatography was performed on Whatman precoated silica gel 60 F-254 plates and visualized by ultra-violet light and/or $10 \%$ phosphomolybdic acid in ethanol with heating. The infrared spectra were obtained using a Perkin-Elmer 1600 series spectrometer. Melting points are reported in degrees Celsius and are uncorrected.

\section{General Procedure A for the Preparation of Tertiary Alcohols. Preparation of 1-} (3-Chlorophenyl)-1-phenyl-1-propanol (S1). The bis(sulfonamide) ligand (5.4 mg, 10

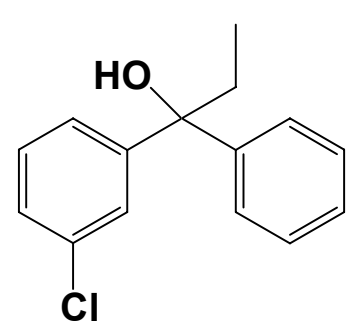
mol\%) was weighed into the well-dried Schlenk flask and put into the dry box. Diphenylzinc $(35.1 \mathrm{mg}, 0.16 \mathrm{mmol})$ was added, previously dissolved in toluene $(1 \mathrm{~mL})$, followed by titanium(IV) isopropoxide $(50 \mu \mathrm{L}, 1.2 \mathrm{M}$ hexanes solution, $0.06 \mathrm{mmol})$. The homogeneous reaction mixture was stirred at room temperature for 15 minutes. 3'-chloropropiophenone $(17.2 \mathrm{mg}, 0.1 \mathrm{mmol})$ was then, added dissolved in toluene $(0.5 \mathrm{~mL})$. The flask was sealed and removed from the dry box. The reaction mixture was stirred at room temperature until TLC showed complete consumption of the ketone. The reaction was quenched with a $15 \%$ aqueous solution of tartaric acid and extracted with $\mathrm{CH}_{2} \mathrm{Cl}_{2}$. The combined organic phases were dried using anhydrous sodium sulfate, filtered and the solvent was removed under vacuum. The residue was purified by silica gel column chromatography (hexanes: EtOAc / $98: 2)$ to give $\mathbf{S 1}(24.4 \mathrm{mg}, 99 \%$ yield, $92 \%$ ee $)$ as an oil: $[\alpha]_{\mathrm{D}}{ }^{20}=+21.4(c$ 
0.81, $\left.\mathrm{CHCl}_{3}\right) ;{ }^{1} \mathrm{H} \mathrm{NMR}\left(\mathrm{CDCl}_{3}, 500 \mathrm{MHz}\right) \delta 0.92(\mathrm{dd}, J=7.3,7.3 \mathrm{~Hz}, 3 \mathrm{H}), 2.12$ (brs, 1H), 2.31-2.38 (m, 2H), 7.22-7.32 (m, 4H), 7.34-7.38 (m, 2H), 7.44-7.46 (m, 2H), 7.50 (brs, 1H) ppm; ${ }^{13} \mathrm{C} \mathrm{NMR}\left(\mathrm{CDCl}_{3}, 125 \mathrm{MHz}\right) \delta 8.45$ (q), 34.8 (t), 78.6 (s), 124.8 (d), 126.4 (d), 126.8 (d), 127.29 (d), 127.5 (d), 128.7 (d), 129.7 (d), 134.5 (s), 146.7 (s), 149.4 (s) ppm; IR (Film) 3562, 3472, 2972, $1446 \mathrm{~cm}^{-1}$; HRMS calcd for $\mathrm{C}_{15} \mathrm{H}_{14} \mathrm{Cl}$ (M $\mathrm{OH})^{+}: 229.07840$, found 229.07902 .

Preparation of 1-(4-Chlorophenyl)-1-phenyl-1-propanol (S2). The general procedure A was applied to 4'-chloropropiophenone (17.4 mg, $0.1 \mathrm{mmol}$ ). The residue

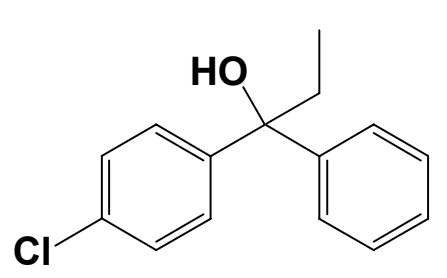
was purified by silica gel column chromatography (hexanes : EtOAc / 98 : 2) to give S2 (24.4 mg, 99\% yield, $88 \%$ ee) as an oil: $[\alpha]_{\mathrm{D}}{ }^{20}=+13.61\left(c 1.0, \mathrm{CHCl}_{3}\right) ;{ }^{1} \mathrm{H}$ $\operatorname{NMR}\left(\mathrm{CDCl}_{3}, 500 \mathrm{MHz}\right) \delta 0.914(\mathrm{dd}, J=7.3,7.3 \mathrm{~Hz}, 3 \mathrm{H})$, 2.29-2.36 (m, 2H), 7.26-7.31 (m, 3H), 7.33-7.39 (m, 4H), 7.42-7.44 (m, 2H) ppm; ${ }^{13} \mathrm{C} \mathrm{NMR}\left(\mathrm{CDCl}_{3}, 125 \mathrm{MHz}\right) \delta 8.4$ (q), 34.8 (t), 78.5 (s), 126.4 (d), 127.4 (d), 128.0 (d), 128.5 (d), 128.6 (d), 132.9 (s), 145.8 (s), 146.9 (s) ppm; IR (Film) 3562, 3462, 2970, $1489 \mathrm{~cm}^{-1}$; HRMS calcd for $\mathrm{C}_{15} \mathrm{H}_{14} \mathrm{Cl}(\mathrm{M}-\mathrm{OH})^{+}$: 229.07840, found 229.07893 .

General Procedure B for the Preparation of Tertiary Alcohols. Preparation of 1-Phenyl-1-(3-trifluoromethylphenyl)-ethanol (S3). The general procedure A was<smiles>CC(O)(c1ccccc1)c1cccc(C(F)(F)F)c1</smiles>
applied to 3'-(trifluoromethyl)acetophenone ( $15 \mu \mathrm{L}, 0.1 \mathrm{mmol})$. Ketone was added neat. The residue was purified by silica gel column chromatography (hexanes : EtOAc / 98 : 2) to give $\mathbf{S 3}$ (24.7 mg, 93\% yield, 95\% ee) as an oil: $[\alpha]_{\mathrm{D}}{ }^{20}=+24.95$ ( c 1.0, $\left.\mathrm{CHCl}_{3}\right) ;{ }^{1} \mathrm{H} \mathrm{NMR}\left(\mathrm{CDCl}_{3}, 500 \mathrm{MHz}\right) \delta 2.01(\mathrm{~s}, 3 \mathrm{H}), 7.29-7.32$ (m, 1H), 7.36-7.39 (m, 2H), 7.43-7.46 (m, 3H), 7.53-7.59 (m, 2H), 6.95-6.97 (m, 4H), 7.80 (brs, 1H) ppm; ${ }^{13} \mathrm{C} \mathrm{NMR}\left(\mathrm{CDCl}_{3}, 125 \mathrm{MHz}\right) \delta 31.2$ (q), 76.3 (s), 115.7 (s), 122.8 (d), 124.1 (d), 126.2 (d), 127.8 (d), 128.8 (d), 128.9 (d), 129.8 (d), 130,0 (s), 147.5 (s), 149.5 (s) ppm; IR (Film) 3429, 1329, 1165, 1124. $\mathrm{cm}^{-1}$; HRMS calcd for $\mathrm{C}_{14} \mathrm{H}_{10} \mathrm{OF}_{3}$ $(\mathrm{M}-\mathrm{Me})^{+}: 251.06837$, found 251.068376. 
Preparation of 1-(2-Bromophenyl)-1-phenylethanol (S4). The general procedure B was applied to 2'-bromoacetophenone $(14 \mu \mathrm{L}, 0.1 \mathrm{mmol})$. The residue was<smiles>CC(O)(c1ccccc1)c1ccccc1Br</smiles>
purified by silica gel column chromatography (hexanes : EtOAc / 98 : 2) to give $\mathbf{S 4}(21.2 \mathrm{mg}, 76 \%$ yield, $95 \%$ ee) as an oil: $[\alpha]_{\mathrm{D}}{ }^{20}=+46.9\left(c 0.89, \mathrm{CHCl}_{3}\right) ;{ }^{1} \mathrm{H} \mathrm{NMR}\left(\mathrm{CDCl}_{3}, 500 \mathrm{MHz}\right) \delta$ $2.01(\mathrm{~s}, 3 \mathrm{H}), 3.50(\mathrm{~s}, 1 \mathrm{H}), 7.21(\mathrm{ddd}, J=1.6,7.8,7.8 \mathrm{~Hz}, 1 \mathrm{H})$, 7.27-7.35 (m, 5H), 7.44 (ddd, $J=1.37 .8,7.8 \mathrm{~Hz}, 1 \mathrm{H}), 7.58$ (dd, $J=1.3,7.8 \mathrm{~Hz}, 1 \mathrm{H})$, $7.86(\mathrm{dd}, J=1.6,7.8 \mathrm{~Hz}, 1 \mathrm{H}) \mathrm{ppm} ;{ }^{13} \mathrm{C} \mathrm{NMR}\left(\mathrm{CDCl}_{3}, 125 \mathrm{MHz}\right) \delta 30.7$ (q), 77.9 (s), 122.7 (s), 126.0 (d), 127.3 (d), 127.7 (d), 128.6 (d), 128.9 (d), 129.5 (d), 135.35 (d), 145.4 (s), 148.0 (s) ppm; IR (Film) 3548, 3442, 2980, $1431 \mathrm{~cm}^{-1}$; HRMS calcd for $\mathrm{C}_{13} \mathrm{H}_{10} \mathrm{OBr}(\mathrm{M}-\mathrm{Me})^{+}: 260.99150$, found 260.99261 .

Preparation of 1-(2-Naphthalenyl)-1-phenylethanol (S5). The general procedure A was applied to 2'-acetonaphthone $(17 \mathrm{mg}, 0.1 \mathrm{mmol})$. The residue was

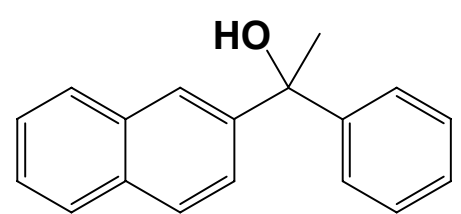
purified by silica gel column chromatography (hexanes : EtOAc / 98 : 2) to give $\mathbf{S 5}(24.5 \mathrm{mg}, 99 \%$ yield, $96 \%$ ee) as an oil: $[\alpha]_{\mathrm{D}}^{20}=+7.07\left(c 1.3, \mathrm{CHCl}_{3}\right)$; ${ }^{1} \mathrm{H}$ NMR $\left(\mathrm{CDCl}_{3}, 500 \mathrm{MHz}\right) \delta 2.09(\mathrm{~s}, 3 \mathrm{H}), 2.34(\mathrm{~s}$, 1H), 7.29-7.31 (m, 1H), 7.35-7.38 (m, 2H), 7.45-7.54 (m, 5H), 7.79-7.89 (m, 3H), 8.02 (s, 1H) ppm; ${ }^{13} \mathrm{C} \mathrm{NMR}\left(\mathrm{CDCl}_{3}, 125 \mathrm{MHz}\right) \delta 31.2$ (q), 76.8 (s), 124.2 (d), 125.3 (d), 126.3 (d), 126.5 (d), 127.4 (d), 127.9 (d), 128.3 (d), 128.6 (d), 128.7 (d), 132.8 (s), 133.4 (s), 145.7 (s), 148.2 (s) ppm; IR (Film) 3440, 3056, 1722, $1445 \mathrm{~cm}^{-1}$; HRMS calcd for $\mathrm{C}_{18} \mathrm{H}_{16} \mathrm{O}(\mathrm{M})^{+}:$248.12011, found 248.12091.

Preparation of 1-(1-Cyclohexenyl)-1-phenylethanol (S6). The general procedure B was applied to 1 -acetyl-1-cyclohexene $(13 \mu \mathrm{L}, 0.1 \mathrm{mmol})$. The residue was purified by<smiles>CC(O)(C1=CCCCC1)c1ccccc1</smiles>
silica gel column chromatography (hexanes : EtOAc / $98: 2$ ) to give S6 (19 mg, 94\% yield, 93\% ee) as an oil: $[\alpha]_{D}{ }^{20}=-16.1(c$ $\left.0.72, \mathrm{CHCl}_{3}\right) ;{ }^{1} \mathrm{H} \mathrm{NMR}\left(\mathrm{CDCl}_{3}, 500 \mathrm{MHz}\right) \delta 1.55-1.62(\mathrm{~m}, 4 \mathrm{H})$, $1.68(\mathrm{~s}, 3 \mathrm{H}), 1.76-1.80(\mathrm{~m}, 1 \mathrm{H}), 1.88(\mathrm{brs}, 1 \mathrm{H}), 1.94-1.98(\mathrm{~m}$, 1H), 2.16-2.17 (m, 2H), 5.93-5.95 (m, 1H) 7.24-7.29 (m, 1H), 7.34- 7.37 (m, 2H), 7.45$7.47(\mathrm{~m}, 1 \mathrm{H}) \mathrm{ppm} ;{ }^{13} \mathrm{C} \mathrm{NMR}\left(\mathrm{CDCl}_{3}, 125 \mathrm{MHz}\right) \delta 22.7(\mathrm{t}), 23.3(\mathrm{t}), 24.9(\mathrm{t}), 25.6(\mathrm{t})$, 29.2 (q), 77.4(s), 122.0 (d), 125.7 (d), 127.0 (d), 128.4 (d), 142.9 (s), 147.3 (s) ppm; IR 
(Film) 3441, 2926, 1446, $1062 \mathrm{~cm}^{-1}$; HRMS calcd for $\mathrm{C}_{14} \mathrm{H}_{18} \mathrm{O}(\mathrm{M})^{+}: 202.13576$, found 202.13654 .

Preparation of 2,4-Diphenyl-3-buten-2-ol (S7). The general procedure A was applied (c) to trans-4-phenyl-3-buten-2-one (14 mg, $0.1 \mathrm{mmol})$. The residue was purified by silica gel column chromatography (hexanes : EtOAc / $98: 2$ ) to give $\mathbf{S 7}$ $\left(13 \mathrm{mg}, 58 \%\right.$ yield, $91 \%$ ee) as an oil: $[\alpha]_{\mathrm{D}}{ }^{20}=+12.8(c$ 0.63, $\left.\mathrm{CHCl}_{3}\right) ;{ }^{1} \mathrm{H} \mathrm{NMR}\left(\mathrm{CDCl}_{3}, 500 \mathrm{MHz}\right) \delta 1.76(\mathrm{~s}, 3 \mathrm{H}), 6.49(\mathrm{~d}, J=16.0 \mathrm{~Hz}, 1 \mathrm{H})$, $6.63(\mathrm{~d}, J=16.0 \mathrm{~Hz}, 1 \mathrm{H}), 7.16-7.38(\mathrm{~m}, 8 \mathrm{H}), 7.51(\mathrm{~d}, J=7.4 \mathrm{~Hz}, 2 \mathrm{H}) \mathrm{ppm} ;{ }^{13} \mathrm{C} \mathrm{NMR}$ $\left(\mathrm{CDCl}_{3}, 125 \mathrm{MHz}\right) \delta 29.8$ (q), 74.6 (s), 125.2 (d), 126.5 (d), 127.0 (d), 127.6 (d), 127.7 (d), 128.3 (d), 128.5 (d), 136.8 (d), 136.7 (s), 146.6 (s) ppm; IR (Film) 3390, 2962, $1710,1493, \mathrm{~cm}^{-1}$; HRMS calcd for $\mathrm{C}_{16} \mathrm{H}_{16} \mathrm{O}(\mathrm{M})^{+}: 224.12011$, found 224.11954.

Preparation of 1-Cyclohexyl-1-phenylethanol (S8). The general procedure B was applied to 1 -cyclohexyl-ethanone $(14 \mu \mathrm{L}, 0.1 \mathrm{mmol})$. The residue was purified by<smiles>CC(O)(c1ccccc1)C1CCCCC1</smiles>
silica gel column chromatography (hexanes : EtOAc / $98: 2$ ) to give S8 (16 mg, $81 \%$ yield, $87 \%$ ee $)$ as an oil: $[\alpha]_{D}{ }^{20}=-16.6(c$ $\left.0.69, \mathrm{CHCl}_{3}\right) ;{ }^{1} \mathrm{H} \mathrm{NMR}\left(\mathrm{CDCl}_{3}, 500 \mathrm{MHz}\right) \delta 0.99-1.21(\mathrm{~m}, 5 \mathrm{H})$ , $1.56(\mathrm{~s}, 3 \mathrm{H}), 1.60-1.79(\mathrm{~m}, 6 \mathrm{H}), 7.24-7.29(\mathrm{~m}, 1 \mathrm{H}), 7.34-7.37$ $(\mathrm{m}, 2 \mathrm{H}), 7.42-7.44(\mathrm{~m}, 2 \mathrm{H}) \mathrm{ppm} ;{ }^{13} \mathrm{C} \mathrm{NMR}\left(\mathrm{CDCl}_{3}, 125 \mathrm{MHz}\right) \delta 26.8(\mathrm{t}), 27.0(\mathrm{t}), 27.0$ (t), $27.2(\mathrm{q}), 27.6$ (t), 27.7(t), 49.4 (d), $77.0(\mathrm{~s}), 125.7$ (d), 126.7 (d), 128.2 (d), 148.3 (s) ppm; IR (Film) 3450, 2923, 1445, $1061 \mathrm{~cm}^{-1}$; HRMS calcd for $\mathrm{C}_{14} \mathrm{H}_{19} \mathrm{O}(\mathrm{M}-\mathrm{H})^{+}$: 203.14359, found 203.14270.

Preparation of 3-Methyl-2-phenyl-2-butanol (S9). The general procedure B was applied to 3-methyl-2-butanone $(21 \mu \mathrm{L}, 0.2 \mathrm{mmol})$. The residue was purified by silica<smiles>CC(C)C(C)(O)c1ccccc1</smiles>
gel column chromatography (hexanes : EtOAc / $98: 2$ ) to give $\mathbf{S 9}$ $\left(18 \mathrm{mg}, 55 \%\right.$ yield, $75 \%$ ee) as an oil: $[\alpha]_{\mathrm{D}}{ }^{20}=+6.0(c \quad 0.05$, $\left.\mathrm{CHCl}_{3}\right) ;{ }^{1} \mathrm{H} \mathrm{NMR}\left(\mathrm{CDCl}_{3}, 500 \mathrm{MHz}\right) \delta 0.84(\mathrm{~d}, J=6.8 \mathrm{~Hz}, 3 \mathrm{H})$, $0.93(\mathrm{~d}, J=6.8 \mathrm{~Hz}, 3 \mathrm{H}), 1.56(\mathrm{~s}, 3 \mathrm{H}), 2.05(\mathrm{dddd}, J=6.8,6.8,13.6$, 13.6 Hz, 1H), 7.24-7.27 (m, 1H), 7.34-7.37 (m, 2H), 7.44-7.46 (m, 2H) ppm; ${ }^{13} \mathrm{C}$ NMR $\left(\mathrm{CDCl}_{3}, 125 \mathrm{MHz}\right) \delta 17.5$ (q), 17.8 (q), 27.1 (q), 39.0 (d), ? (s), 125.6 (d), 126.8 (d), 
128.2 (d), 148.2 (s) ppm; IR (Film) 2924, 2358, 1736, $1095 \mathrm{~cm}^{-1}$; HRMS calcd for $\mathrm{C}_{11} \mathrm{H}_{15}(\mathrm{M}-\mathrm{OH})^{+}:$147.11737, found 147.11784.

Preparation of 2-Bromo-1-(2-naphthalenyl)-1-phenylethanol (S10). The general procedure A was applied to $\alpha$-bromo-2'-acetonaphthone (102 mg, $0.4 \mathrm{mmol})$.

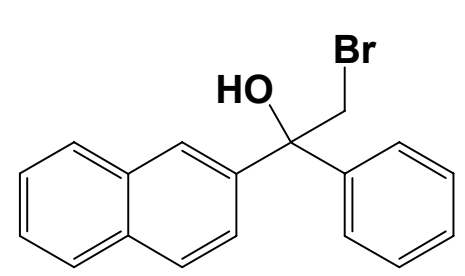

The residue was purified by silica gel column chromatography (hexanes : EtOAc / $98: 2$ ) to give $\mathbf{S 1 0}$ (86 mg, 66\% yield, $79 \%$ ee) as an oil: $[\alpha]_{\mathrm{D}}^{20}=+2.64(c$ 2.04, $\left.\mathrm{CHCl}_{3}\right) ;{ }^{1} \mathrm{H} \mathrm{NMR}\left(\mathrm{CDCl}_{3}, 500 \mathrm{MHz}\right) \delta 3.25(\mathrm{~s}$, $1 \mathrm{H}), 4.24(\mathrm{~d}, J=11 \mathrm{~Hz}, 1 \mathrm{H}), 4.28(\mathrm{~d}, J=11 \mathrm{~Hz}, 1 \mathrm{H})$, 7.32-7.40 (m, 3H), 7.51-7.56 (m, 5H), 7.84-7.89 (m, 3H), $8.01(\mathrm{~s}, 1 \mathrm{H}) \mathrm{ppm} ;{ }^{13} \mathrm{C} \mathrm{NMR}$ $\left(\mathrm{CDCl}_{3}, 125 \mathrm{MHz}\right) \delta 44.2$ (d), 77.4 (s), 124.9 (d), 125.6 (d), 126.7 (d), 126.7 (d), 126.8 (d), 127.9 (d), 128.2 (d), 128.6 (d), 128.8 (d), 128.8 (s), 133.1 (s), 133.4 (s), 141.1 (s), 143.6 (s) ppm; IR (Film) 3538, 3056, 2924, $1679 \mathrm{~cm}^{-1}$; HRMS calcd for $\mathrm{C}_{18} \mathrm{H}_{15} \mathrm{OBr}$ $(\mathrm{M})^{+}: 326.03062$, found 326.03077 .

Preparation of 2-(2-Naphthalenyl)-2-phenyloxirane (S11). To the 2-Bromo-1(2-naphthalenyl)-1-phenylethanol (S10) $(21 \mathrm{mg}, 0.06 \mathrm{mmol})$ in acetonitrile $(1 \mathrm{~mL})$ were<smiles>c1ccc(C2(c3ccc4ccccc4c3)CO2)cc1</smiles>
successively added benzylamine $(50 \mu \mathrm{L}, 0.45 \mathrm{mmol})$ and $\mathrm{LiClO}_{4} \cdot 3 \mathrm{H}_{2} \mathrm{O}(50 \mathrm{mg}, 0.312 \mathrm{mmol})$ at room temperature. After stirring at room temperature for $2 \mathrm{~h}$, water was added, and the mixture was extracted with ethyl acetate.

The combined organic layers were washed with brine, and dried over anhydrous $\mathrm{Na}_{2} \mathrm{SO}_{4}$. The solvent was evaporated, and the residue was purified by silica gel column chromatography (hexanes : EtOAc / $98: 2$ ) to give $\mathbf{S 1 1 ~ ( 1 4 ~ m g , ~ 9 0 \% ~ y i e l d , ~ 7 8 \% ~ e e ) ~ a s ~}$ an oil: $[\alpha]_{\mathrm{D}}{ }^{20}=-22.6\left(c 0.64, \mathrm{CH}_{3} \mathrm{OH}\right) ;{ }^{1} \mathrm{H}$ NMR $\left(\mathrm{C}_{6} \mathrm{D}_{6}, 500 \mathrm{MHz}\right) \delta 2.90(\mathrm{~d}, J=5.5$ $\mathrm{Hz}, 1 \mathrm{H}), 2.93(\mathrm{~d}, J=5.5 \mathrm{~Hz}, 1 \mathrm{H}), 7.04-7.18(\mathrm{~m}, 5 \mathrm{H}), 7.30-7.31(\mathrm{~m}, 2 \mathrm{H}), 7.38-7.39(\mathrm{~m}$, $1 \mathrm{H}), 7.49-7.52(\mathrm{~m}, 3 \mathrm{H}), 7.75(\mathrm{brs}, 1 \mathrm{H}) \mathrm{ppm} ;{ }^{13} \mathrm{C} \mathrm{NMR}\left(\mathrm{C}_{6} \mathrm{D}_{6}, 125 \mathrm{MHz}\right) \delta 56.2(\mathrm{t}), 61.6$ (s), 125.3 (d), 126.0 (d), 126.1 (d), 126.8 (d), 127.5 (d), 127.6 (d), 127.7 (d), 128.0 (d), 128.0 (d), 128.1 (d), 133.1 (s), 133.2 (s), 137.4 (s), 140.0 (s) ppm; IR (Film) 3055, 2923, 1494, $1296 \mathrm{~cm}^{-1}$; HRMS calcd for $\mathrm{C}_{18} \mathrm{H}_{14} \mathrm{O}(\mathrm{M})^{+}:$246.10446, found 246.10415. 


\section{Conditions for the Determination of Enantiomeric Excess.}

The racemic alcohols were prepared by addition of phenylmagnesium bromide to the corresponding ketone. Only the tertiary alcohol S9 were analyzed by chiral capillary GC. The specifications for the GC analyses were as follows: Fused silica chiral capillary column (Supelco $\beta$-Dex 120): $30 \mathrm{~m}$ x $0.25 \mathrm{~mm}$ (id) x $0.25 \mu \mathrm{m}$ film

thickness. Carrier gas: nitrogen. Inlet temperature: $250{ }^{\circ} \mathrm{C}$. Detector: FID, $270{ }^{\circ} \mathrm{C}$. The conditions for the resolution of the racemates by $\mathrm{GC}$ are given below.

3-Methyl-2-phenyl-2-butanol (S9). $\mathrm{t}_{1}=36.7 \mathrm{~min}, \mathrm{t}_{2}=38.6 \mathrm{~min}\left(115^{\circ} \mathrm{C}, 1.0 \mathrm{~mL} / \mathrm{min}\right)$.

Chiral HPLC analyses of S1 - S8, and S10 - S11 were performed using a Chiralcel OD-H column. The conditions for the resolution of the racemates are described below.

1-(3-Chlorophenyl)-1-phenyl-1-propanol (S1). $\mathrm{t}_{1}=19.5 \mathrm{~min}, \mathrm{t}_{2}=22.4 \mathrm{~min}$ (hexane $/$ 2-propanol : 92 / 8, $0.5 \mathrm{~mL} / \mathrm{min})$.

1-(4-Chlorophenyl)-1-phenyl-1-propanol (S2). $\mathrm{t}_{1}=26.9 \mathrm{~min}, \mathrm{t}_{2}=30.7 \mathrm{~min}$ (hexane $/$ 2-propanol : 95 / 5, $0.5 \mathrm{~mL} / \mathrm{min})$.

1-Phenyl-1-(3-trifluoromethylphenyl)-ethanol (S3). $t_{1}=25.0 \mathrm{~min}, t_{2}=28.3 \mathrm{~min}$ (hexane / 2-propanol : 95 / 5, $0.5 \mathrm{~mL} / \mathrm{min}$ ).

1-(2-Bromophenyl)-1-phenylethanol (S4). $t_{1}=15.3 \mathrm{~min}, \mathrm{t}_{2}=17.1 \mathrm{~min}$ (hexane $/ 2$ propanol : 95 / 5, $0.5 \mathrm{~mL} / \mathrm{min})$.

1-(2-Naphthalenyl)-1-phenylethanol (S5). $\mathrm{t}_{1}=35.1 \mathrm{~min}, \mathrm{t}_{2}=40.3 \mathrm{~min}$ (hexane $/ 2$ propanol : 95 / 5, $0.5 \mathrm{~mL} / \mathrm{min})$.

2,4-Diphenyl-3-buten-2-ol (S6). $\mathrm{t}_{1}=15.6 \mathrm{~min}, \mathrm{t}_{2}=20.6 \mathrm{~min}$ (hexane / 2-propanol : 92 / $8,0.8 \mathrm{~mL} / \mathrm{min})$.

1-(1-Cyclohexenyl)-1-phenylethanol (S7). $t_{1}=17.5 \mathrm{~min}, \mathrm{t}_{2}=20.0 \mathrm{~min}$ (hexane $/ 2$ propanol : $98 / 2,0.5 \mathrm{~mL} / \mathrm{min})$.

1-Cyclohexyl-1-phenylethanol (S8). $\mathrm{t}_{1}=12.6 \mathrm{~min}, \mathrm{t}_{2}=15.4 \mathrm{~min}$ (hexane / 2-propanol : $95 / 5,0.5 \mathrm{~mL} / \mathrm{min})$.

2-Bromo-1-(2-naphthalenyl)-1-phenylethanol (S10). $\mathrm{t}_{1}=62.1 \mathrm{~min}, \mathrm{t}_{2}=66.4 \mathrm{~min}$ (hexane / 2-propanol : 99 / 1, $0.9 \mathrm{~mL} / \mathrm{min}$ ). 
2-(2-Naphthalenyl)-2-phenyloxirane (S11). $\mathrm{t}_{1}=12.1 \mathrm{~min}, \mathrm{t}_{2}=15.1 \mathrm{~min}$ (hexane $/ 2$ propanol : 95 / 5, $0.5 \mathrm{~mL} / \mathrm{min}$ ). 